\title{
Review of: "Architecting functionalized carbon microtube/carrollite nanocomposite demonstrating significant microwave characteristics"
}

\author{
Renchao $\mathrm{Che}^{1}$ \\ 1 Fudan University
}

Potential competing interests: The author(s) declared that no potential competing interests exist.

Recommendation: minor revision

Comments: In this work, the authors report the synthesis of carbon microtube/carrollite nanocomposite and their microwave absorption, EMI shielding, and antibacterial applications. The results are clear and well organized. The work is meaningful. Therefore, I recommend its publication after some minor revisions. Detailed comments include the follows:

(1) It makes me confused that imaginary of permittivity of CuCo2S4/PAN is negative in high frequency, while strong resonance peak in its imaginary permeability appears at almost the same frequency. I suggest the author recheck measurement. The detailed test procedure should be listed.

(2) The origins of EM loss have been investigated with a unique electron holography technology, which may help the author to enrich the related mechanism part. The recommended references: Adv. Mater. 2004, 16, 401; Adv. Mater. 2014, 26, 8120; Adv. Mater. 2016, 28, 486-490; Small. 2012, 8, 1214; Appl. Phys. Lett. 2006, 88, 033105; Adv. Funct. Mater. 2019, 29, 1901448.

(3) According to microwave absorption principle, it would be better to simultaneously provide loss capacity in dielectric and magnetic ways. So, why does the author choose metal sulfide rather than their magnetic oxides? 